\title{
A Review of Marital Intimacy-Enhancing Interventions among Married Individuals
}

\author{
Maryam Kardan-Souraki ${ }^{1,2}$, Zeinab Hamzehgardeshi ${ }^{3,4}$, Ismail Asadpour ${ }^{5}$, Reza Ali Mohammadpour ${ }^{6} \&$ Soghra $^{3}$ \\ $\mathrm{Khani}^{3,7}$ \\ ${ }^{1}$ Faculty of Nasibeh Nursing and Midwifery, Mazandaran University of Medical Sciences, Sari, Iran \\ ${ }^{2}$ Student Research Committee, Mazandaran University of Medical Sciences, Sari, Iran \\ ${ }^{3}$ Department of Midwifery and Reproductive Health, Faculty of Nasibeh Nursing and Midwifery, Mazandaran \\ University of Medical Sciences, Sari, Iran \\ ${ }^{4}$ Research Center of Traditional Medicine, Mazandaran University of Medical Sciences, Sari, Iran \\ ${ }^{5}$ Department of Counseling, Faculty of Psychology and Educational Sciences, Kharazmi University, Karaj, Iran \\ ${ }^{6}$ Department of Biostatistics, Faculty of Health, Mazandaran University of Medical Sciences, Sari, Iran \\ ${ }^{7}$ Research Center of Diabetes, Mazandaran University of Medical Sciences, Sari, Iran \\ Correspondence: Soghra Khani, Department of Midwifery and Reproductive Health, Nasibeh Nursing and \\ Midwifery Faculty, Mazandaran University of Medical Sciences, Sari, Iran. Tel: 98-11-3336-8916. E-mail: \\ s.khani@mazums.ac.ir; khanisog343@gmail.com
}

Received: September 12, 2015 Accepted: November 21, 2015 Online Published: December 17, 2015

doi:10.5539/gjhs.v8n8p74

URL: http://dx.doi.org/10.5539/gjhs.v8n8p74

\begin{abstract}
Background: Lack of intimacy is currently the main concern rather than main concern of the experts in psychology and counseling. It is considered as one of the most important causes for divorce and as such to improve marital intimacy a great number of interventions have been proposed in the literature. Intimacy training and counseling make the couples take effective and successful steps to increase marital intimacy. No study has reviewed the interventions promoting marital intimacy after marriage. Thus, this review study aimed to classify the articles investigating the impact of interventional programs on marital intimacy after marriage.
\end{abstract}

Search Methods: In April 2015, we performed a general search in Google Scholar search engines, and then we did an advanced search the databases of Science Direct, ProQuest, SID, Magiran, Irandoc, Pubmed, Scopus, Cochrane Library, and Psych info; Cumulative Index to Nursing and Allied Health Literature (CINAHL). Also, lists of the references of the relevant articles were reviewed for additional citations. Using Medical Subject Headings (MESH) keywords: Intervention (Clinical Trials, Non-Randomized Controlled Trials, Randomized Controlled Trials, Education), intimacy, marital (Marriage) and selected related articles to the study objective were from 1995 to April 2015. Clinical trials that evaluated one or more behavioral interventions to improve marital intimacy were reviewed in the study.

Main Results: 39 trials met the inclusion criteria. Eleven interventions had follow-up, and 28 interventions lacked follow-up. The quality evidence for 22 interventions was low, for 15 interventions moderate, and for one intervention was considered high. Findings from studies were categorized in 11 categories as the intimacy promoting interventions in dimensions of emotional, psychological, physical, sexual, temporal, communicational, social and recreational, aesthetic, spiritual, intellectual intimacy, and total intimacy.

Authors' Conclusions: Improving and promoting communication, problem solving, self-disclosure and empathic response skills and sexual education and counseling in the form of cognitive-behavioral techniques and based on religious and cultural context of each society, an effective step can be taken to enhance marital intimacy and strengthen family bonds and stability. Health care providers should consider which interventions are appropriate to the couple characteristics and their relationships.

Keywords: intervention, intimacy, marital 


\section{Introduction}

Marriage is a transient phase in one's life and has always been emphasized as the paramount social ritual in order to meet the emotional needs of people (Dildar, Sitwat, \& Yasin, 2013; Nayeri, Lotfi, \& Noorani, 2014). In contemporary society, the incentives to marriage include the need to love and have intimate relationship with a partner, to have a companion in life, to satisfy psychological needs, and to increase joy (Soltani, Molazadeh, Mahmoodi, \& Hosseini, 2013; Tavakol, Zarei, \& Zeinali Pour, 2014).

Intimacy includes different meanings based on age, sex, education, and culture, and there is no consensus among researchers on the root concept of intimacy which makes its definition difficult (Martin \& Tardif, 2014; Mitchell, 2007). Bagarozzi (2001) defines intimacy as proximity, similarity and a personal romantic or emotional communication that requires knowledge and understanding of another person to express thoughts and feelings (Bagarozzi, 2001).

Intimacy is strongly associated with the quality of couples' life and is often referred to as a basic psychological need and one of the key characteristics of marital communication which impacts on marital adjustment and mental health, such as reducing the risk of depression, increasing happiness and well-being, and providing a useful satisfactory life of a person. Besides, it is a strong predictor of physical health, such as low level of diseases and impoverishment of diseases (Boden, Fischer, \& Niehuis, 2010; Dandurand \& Lafontaine, 2013; Moreira, Crespo, Pereira, \& Canavarro, 2010; Nainian \& Nik-Azin, 2013). In a study was shown that marital intimacy is effective on marital satisfaction (Greeff, Hildegarde, \& Malherbe, 2001; Kim, 2013). Intimacy acts as a mediator between the effects of daily stress in relations between spouses (Harper, Schaalje, \& Sandberg, 2000). There is a significant positive correlation between sexual satisfaction and marriage commitment with intimacy (Taghiyar, Mohammadi, \& Zarie, 2015). In contrast, lack of intimacy is one of the most common causes of distress and collapse among couples, negatively impacting on relations between the couples and, thereby, leading to incompatibility and causes stress, and brings about psychological maladaptation, depression, and emotional disorders mental disorders (Duffey, Wooten, Lumadue, \& Comstock, 2004; Kim, 2013; Yoo, Bartle-Haring, Day, \& Gangamma, 2014). Dearth of intimacy is one of the most devastating problems that is difficult to be treated in the relationships (Whisman, Dixon, \& Johnson, 1997). Weinberger et al. (2008) also showed that lack of intimacy in couples is the most important predictor of divorce in elderly (Weinberger, Hofstein, \& Whitbourne, 2008). Thus, it can be stated that the consequences of failure in intimacy are manifold and physical divorce mainly arises from failure in intimacy (Duffey et al., 2004).

Therapists have described on various aspects that may negatively influence marital stability such as communication difficulty, unrealistic expectations from marriage and the spouse, lack of intimacy, and lack of expressing affection (Motavali, Ozgoli, Bakhtiari, \& Alavimajd, 2010; Shahrestany, Doustkam, Rahbarda, \& Mashhadi, 2013). Taking the fact for the granted that in many societies today family is the prominent source of comfort for people and taking the fact that in the modern society the family is faced with the challenges, the most important of which is the loss of marital intimacy, into account, an interventional program is helpful to prevent these problems and heighten intimacy (Farbod, Ghamari, \& Majd, 2014). To enhance () intimacy in couples, educational approaches may support () (Oulia, Fatehizadeh, \& Bahrami, 2006). It believed that Education and counseling per se may make the couples take effective and successful steps to increase marital intimacy (Hosseini Zand, SHafi Abadi, \& Soudani, 2013). In Iran, some interventions are done to increase marital intimacy. For example, In a study was shown that training communication skills can enhance intimacy and quality in marital life (Farbod et al., 2014). Moreover, KhanjaniVeshki et al. (2012) concluded that sex education is effective in increasing sexual intimacy (Khanjani Veshki, Botlani, Shahsiah, \& Sharifi, 2012). Duffey et al. (2004) also showed that sharing dreams and events between couples contributes to an increase in intimacy in couples (Duffey et al., 2004). According to the researcher's search in the databases available, no study has reviewed the interventions promoting marital intimacy after marriage thus far. To address the latter the aim of this review is to classify the articles investigating the impact of interventional programs on marital intimacy after marriage.

\section{Method}

\subsection{The Criteria Considered for This Review}

\subsubsection{Type of Study}

Clinical trials that evaluated one or more behavioral interventions to improve marital intimacy were reviewed in the study. Trials that focused on people with drug abuse and chronic health conditions, such as cancer, were excluded. The reason for their exclusion pertains to statistical population. This is because training and counseling to them were not proportionate to type of disorder in individuals and may not be applied for all spouses. Like 
educations and counselings that focused on people with breast cancer or prostate cancer. There was no other exclusion criterion.

\subsubsection{Type of Participants}

Married men and women or couples.

\subsubsection{Type of Interventions}

Interventions can have different formats such as verbal communication or written methods, individual or group counseling as well as using different types of technology, such as providing educational CDs. Intervention can be provided in a clinic or in the community and can target men, women, or couples. The comparison can be performed between the intervention under the study and another behavioral intervention, usual care or without any intervention.

\subsubsection{Type of the Measured Result}

Our interest outcome was increasing intimacy. All trials that yielded this result were incorporated in the study.

\subsubsection{Type of Intimacy Assessment Tools}

To evaluate interventions, different intimacy questionnaires can be applied such as:

Marital Intimacy Questionnaire Thompson and Walker Marital Intimacy Questionnaire (MIQ) (den Broucke \& Vertommen (1995), Waring Intimacy Questionnaire (WIQ), Personal Assessment of Intimacy in Relationships inventory, Oulia's Couples intimacy questionnaire, and Bagarozzi's Marital Intimacy Needs Questionnaire.

\subsection{Search Method}

In April 2015, we performed a general search in Google Scholar search engines followed by an advanced search was done in the below databases:

ProQuest, Science Direct, SID, Irandoc, Magiran, Pubmed, Cochrane Library, Scopus, and Psych info; Cumulative Index to Nursing and Allied Health Literature (CINAHL).

Keywords were arranged based on Medical Subject Headings (MeSH) to search in Medline and based on non-mesh keywords in other databases including: Intervention (Clinical Trials, Non-Randomized Controlled Trials, Randomized Controlled Trials, and Education), intimacy and marital (Marriage).

Also, lists of the references of the relevant articles were reviewed for additional citations. Selected related articles to the study objective were from 1995 to April 2015.

\subsection{Interventions Quality}

The quality of evidence was evaluated. At first, the quality of the intervention design, implementation, and reports was evaluated. Quality of intervention downgraded for each of the following studies: 1) implementing intervention in less than two sessions , 2) the accuracy of reported interventional information for fewer than three items (Table 1), and 3) lack of follow-up (Lopez, Hiller, Grimes, \& Chen, 2012; Lopez, Steiner, Grimes, \& Schulz, 2013). The quality of the interventions evidence was recorded (Table 2) among the overall assessments of the quality of evidence (Table 3), the quality trials were considered high, then in the case of any of the following, one level of the quality of evidence was downgraded, A) lack of information on random sequence, allocation concealed, or lack of allocation concealed B) low quality interventions, and c) loss of more than $20 \%$ at follow-up. We considered a positive level for the studies that performed blinding procedures (Lopez et al., 2012).

Table 1. Intervention fidelity information

\begin{tabular}{lllll}
\hline Study & $\begin{array}{l}\text { Provider } \\
\text { credentials }\end{array}$ & $\begin{array}{l}\text { Provider } \\
\text { education }\end{array}$ & Standardized delivery & $\begin{array}{l}\text { Delivery } \\
\text { adherence }\end{array}$ \\
\hline Hosseinian (2012) & -----* & ---- & $\begin{array}{l}1.5 \text { hour session of } \\
\text { communication skill based } \\
\text { on Miller Theory }\end{array}$ \\
Asadpour (2012) & Consultant & ------ & $\begin{array}{l}12 \text { sessions of emotionally by step and } \\
\text { stong with weekly } \\
\text { focused couple therapy that assignments and } \\
\text { each session lasted 2/5 hours regular exercises } \\
\text { provided by }\end{array}$ \\
\hline
\end{tabular}




\begin{tabular}{|c|c|c|c|c|}
\hline Study & $\begin{array}{l}\text { Provider } \\
\text { credentials }\end{array}$ & $\begin{array}{l}\text { Provider } \\
\text { education }\end{array}$ & Standardized delivery & $\begin{array}{l}\text { Delivery } \\
\text { adherence }\end{array}$ \\
\hline & & & & consultants \\
\hline Zarepour (2010) & ---- & ----- & $\begin{array}{l}\text { The structure of sessions and } \\
\text { trainings materials presented } \\
\text { at each session were taken } \\
\text { from Davison and Goldfried, } \\
\text { Jacobson and Margolin, } \\
\text { Miller et al., Bernstein and } \\
\text { Bernstein, and was } \\
\text { introduced during } 6 \text { weeks } \\
\text { of one hour sessions }\end{array}$ & $\begin{array}{l}\text { Training sessions } \\
\text { were presented } \\
\text { based on training } \\
\text { curriculum }\end{array}$ \\
\hline Salimi (2012) & ----- & ----- & $\begin{array}{l}\text { sex education was presented } \\
\text { in cognitive behavioral } \\
\text { method during } 6 \text { sessions } \\
\text { each lasted two hours }\end{array}$ & $\begin{array}{l}\text { Training sessions } \\
\text { were presented } \\
\text { based on training } \\
\text { curriculum }\end{array}$ \\
\hline Nasr Isfahani (2013) & ----- & ----- & $\begin{array}{l}\text { meaning - focused workshop } \\
\text { in } 10 \text { sessions of } 90 \text { minutes }\end{array}$ & $\begin{array}{l}\text { Once a week and } \\
\text { based on the } \\
\text { curriculum }\end{array}$ \\
\hline Etemadi (2006) & consultant & ----- & $\begin{array}{l}10 \text { sessions of one-hour } \\
\text { couple therapy based on } \\
\text { cognitive } \\
\text { techniques }\end{array}$ & $\begin{array}{l}\text { Step by step and } \\
\text { though weekly } \\
\text { assignments }\end{array}$ \\
\hline Ebrahimi (2011) & ---- & ----- & $\begin{array}{l}\text { Communication enrichment } \\
\text { program during } 10 \text { sessions } \\
\text { of } 1.5 \text { hours }\end{array}$ & $\begin{array}{l}\text { One session in a } \\
\text { week }\end{array}$ \\
\hline Rezaei (2013) & researcher & ----- & $\begin{array}{l}7 \text { sessions of Islamic } \\
\text { lifestyle training with an } \\
\text { emphasis on the family } \\
\text { system }\end{array}$ & $\begin{array}{l}\text { Twice a week } \\
\text { sessions for } 90 \\
\text { minutes each time }\end{array}$ \\
\hline Shakarami (2014) & ----- & ----- & $\begin{array}{l}6 \text { sessions of two hours sex } \\
\text { education in the form of } \\
\text { speech, asking questions, } \\
\text { group discussion and } \\
\text { presentation of assignments }\end{array}$ & $\begin{array}{l}\text { Weekly program } \\
\text { and based on the } \\
\text { curriculum }\end{array}$ \\
\hline Ghadam kheir (2013) & ----- & ----- & $\begin{array}{l}8 \text { sessions of intervention } \\
\text { based } \\
\text { intellectual-emotional } \\
\text { behavior therapy }\end{array}$ & $\begin{array}{l}\text { For eight weeks, } \\
\text { every week for an } \\
\text { hour and a half in } \\
\text { groups }\end{array}$ \\
\hline Mazlomi (2012) & ----- & ----- & $\begin{array}{l}\text { Marriage } \\
\text { preventive enrichment } \\
\text { designed by } \text { program } \\
\text { presented during } 7 \text { weeks and }\end{array}$ & $\begin{array}{l}\text { Every week one } \\
\text { communication } \\
\text { skill was taught to } \\
\text { couples. }\end{array}$ \\
\hline Etemadi (2014) & ----- & ----- & $\begin{array}{l}\text { Eight sessions of an hour } \\
\text { and a half of group training } \\
\text { based on communication } \\
\text { therapy approach }\end{array}$ & $\begin{array}{l}\text { Weekly program } \\
\text { and based on the } \\
\text { curriculum }\end{array}$ \\
\hline Hosseini Zand (2013) & researcher & ----- & $\begin{array}{l}10 \text { sessions of two hours of } \\
\text { Islamic couple therapy } \\
\text { training }\end{array}$ & $\begin{array}{l}\text { Implemented once } \\
\text { a week and in } \\
\text { three stages }\end{array}$ \\
\hline Shariatzadeh (2014) & researcher & ----- & $\begin{array}{l}10 \text { training sessions based } \\
\text { on choice theory }\end{array}$ & ----- \\
\hline Oulia (2006) & ----- & ----- & $\begin{array}{l}6 \text { sessions of } 90 \text {-minute of } \\
\text { marital life enrichment } \\
\text { training }\end{array}$ & $\begin{array}{l}\text { Sessions were } \\
\text { hold step by step } \\
\text { and weekly }\end{array}$ \\
\hline BabaeiGarmkhani(2014) & ----- & ----- & $\begin{array}{l}8 \text { sessions of } 90 \text {-minute of } \\
\text { cognitive behavioral group } \\
\text { training }\end{array}$ & $\begin{array}{l}\text { Twice a week and } \\
\text { based on the } \\
\text { curriculum }\end{array}$ \\
\hline
\end{tabular}




\begin{tabular}{|c|c|c|c|c|}
\hline Study & $\begin{array}{l}\text { Provider } \\
\text { credentials }\end{array}$ & $\begin{array}{l}\text { Provider } \\
\text { education }\end{array}$ & Standardized delivery & $\begin{array}{l}\text { Delivery } \\
\text { adherence }\end{array}$ \\
\hline Botlani (2010) & ----- & ----- & $\begin{array}{l}8 \text { attachment-based couple } \\
\text { therapy sessions }\end{array}$ & $\begin{array}{l}\text { Once a week and } \\
\text { each session } 90 \\
\text { minutes }\end{array}$ \\
\hline Hosseini (2013) & ----- & ----- & $\begin{array}{l}8 \text { sessions of } 90 \text {-minute of } \\
\text { solution-focused group } \\
\text { counseling }\end{array}$ & $\begin{array}{l}\text { Weekly and based } \\
\text { on the curriculum }\end{array}$ \\
\hline Nasr Isfahani (2010) & ----- & ----- & $\begin{array}{l}7 \text { sessions of } 90 \text { minute of } \\
\text { teaching concepts of choice } \\
\text { theory }\end{array}$ & $\begin{array}{l}\text { Once a week and } \\
\text { based on the } \\
\text { curriculum }\end{array}$ \\
\hline Bahrami (2009) & $\begin{array}{l}\text { offered } \\
\text { under the } \\
\text { counseling } \\
\text { and leading } \\
\text { of the } \\
\text { supervising } \\
\text { professor }\end{array}$ & ----- & $\begin{array}{l}\text { the } 6 \text { session } 2-\mathrm{hr} \text { group } \\
\text { enrichment program training }\end{array}$ & Weekly \\
\hline Durana (1997) & therapist & $\begin{array}{l}\text { Leaders are } \\
\text { license mental } \\
\text { health } \\
\text { professional }\end{array}$ & $\begin{array}{l}\text { The standard 4-month } \\
\text { PAIRS format }\end{array}$ & $\begin{array}{l}\text { Weekly or } \\
\text { biweekly } 3 \text { hour } \\
\text { sessions and } 4 \text { or } \\
5 \text { weekend } \\
\text { workshop lasting } \\
\text { about } 21 \text { hours. }\end{array}$ \\
\hline Khanjani veshki (2012) & counselor & ----- & $\begin{array}{l}6 \text { sessions of sex education. } \\
\text { Format and content } \\
\text { identified for sessions }\end{array}$ & $\begin{array}{l}\text { Sex training was } \\
\text { presented step by } \\
\text { step for men and } \\
\text { women }\end{array}$ \\
\hline Duffey (2004) & researcher & ---- & $\begin{array}{l}\text { offering of the } \\
\text { intimacy-building } \\
\text { dream-sharing workshop } \\
\text { and workbook used to the } \\
\text { interventional group an } \\
\text { intimacy-building and } \\
\text { event-sharing workshop } \\
\text { presented to the control } \\
\text { treatment group }\end{array}$ & $\begin{array}{l}\text { a four hour dream } \\
\text { sharing workshop }\end{array}$ \\
\hline Nayari (2014) & ----- & ----- & $\begin{array}{l}8 \text { sessions of Transactional } \\
\text { Analysis that each session } \\
\text { lasted } 1 / 5 \text { hours }\end{array}$ & $\begin{array}{l}\text { Training sessions } \\
\text { were presented } \\
\text { based on training } \\
\text { curriculum }\end{array}$ \\
\hline Hajian (2013) & ----- & ---- & $\begin{array}{l}\text { An intensive course of } \\
\text { solution-focused couples } \\
\text { therapy was presented } \\
\text { within six sessions that each } \\
\text { session lasts } 1.5 \text { hours }\end{array}$ & $\begin{array}{l}\text { Sessions for } 2.6 \\
\text { months with } \\
\text { giving } \\
\text { assignments and } \\
\text { feedbacks that } \\
\text { presented based } \\
\text { on Objectives } \\
\text { listed for each } \\
\text { visit. }\end{array}$ \\
\hline Nasirnejhad karaj(2014) & ----- & ----- & $\begin{array}{l}\text { Training positive thinking } \\
\text { skills during } 8 \text { sessions that } \\
\text { each session lasted } 1 / 5 \\
\text { hours. Format and content } \\
\text { identified for sessions }\end{array}$ & Weekly sessions \\
\hline Mami (2015) & consultant & ----- & $\begin{array}{l}\text { ten sessions of } 60 \text { minutes } \\
\text { couples therapy and } \\
\text { cognitive-behavioral } \\
\text { techniques }\end{array}$ & Weekly sessions \\
\hline
\end{tabular}




\begin{tabular}{|c|c|c|c|c|}
\hline Study & $\begin{array}{l}\text { Provider } \\
\text { credentials }\end{array}$ & $\begin{array}{l}\text { Provider } \\
\text { education }\end{array}$ & Standardized delivery & $\begin{array}{l}\text { Delivery } \\
\text { adherence }\end{array}$ \\
\hline Farbod (2014) & ----- & ----- & $\begin{array}{l}12 \text { sessions to enhance } \\
\text { communication skills based } \\
\text { marriage and family therapy }\end{array}$ & No information \\
\hline Mohamadi (2013) & ----- & ----- & ---- & ----- \\
\hline Coutta (2002) & A couple & $\begin{array}{l}\text { A couple } \\
\text { trained and } \\
\text { husband had a } \\
\text { Divinity degree } \\
\text { with an } \\
\text { emphasis in } \\
\text { psychology and } \\
\text { counseling. }\end{array}$ & $\begin{array}{l}\text { A weekend marriage } \\
\text { enrichment program With } \\
\text { emphasis on Integrative } \\
\text { Couple Therapy (ICT) } \\
\text { developed by Neil Jacobson } \\
\text { and Andrew Christensen. }\end{array}$ & $\begin{array}{l}\text { Training session } \\
\text { were presented } \\
\text { based on training } \\
\text { curriculum }\end{array}$ \\
\hline $\begin{array}{l}\text { Nasirnejhad [Tehran] } \\
(2014)\end{array}$ & ----- & ----- & $\begin{array}{l}8 \text { sessions of } 1.5 \text { hours } \\
\text { training positive thinking } \\
\text { skills. Format and content } \\
\text { identified for sessions }\end{array}$ & twice a week \\
\hline Sharifian (2011) & ----- & ----- & $\begin{array}{l}12 \text { sessions of couple } \\
\text { communication program } \\
(\mathrm{CCP}) \text { that each session } \\
\text { lasted } 2 \text { hours }\end{array}$ & once a week \\
\hline \multirow{2}{*}{ Hickmon (1997) } & \multirow{2}{*}{$\begin{array}{l}\text { first author } \\
\text { and a couple }\end{array}$} & $\begin{array}{l}\text { The husband } \\
\text { was in his final } \\
\text { week of a } \\
\text { masters' degree } \\
\text { program in }\end{array}$ & \multirow{2}{*}{$\begin{array}{l}\text { In the Adventure group, } \\
\text { Waring's (1984) } 8 \\
\text { components of marital } \\
\text { intimacy in the design was } \\
\text { used }\end{array}$} & \multirow{2}{*}{ two-day weekend } \\
\hline & & $\begin{array}{l}\text { Bible and } \\
\text { Religion, } \\
\text { training to be a } \\
\text { family life } \\
\text { minister. }\end{array}$ & & \\
\hline Soltani (2013) & ----- & ----- & $\begin{array}{l}8-10 \text { sessions } 120 \text { minutes of } \\
\text { emotionally focused couple } \\
\text { therapy( EFCT) }\end{array}$ & $\begin{array}{l}\text { EFCT has } 3 \text { stages } \\
\text { and } 9 \text { steps. }\end{array}$ \\
\hline Denton (2000) & therapist & $\begin{array}{l}\text { Therapist was } \\
\text { provided with } \\
12 \text { hr of } \\
\text { training in } \\
\text { emotion } \\
\text { focused therapy } \\
\text { that covered the } \\
\text { theory and } \\
\text { techniques of } \\
\text { the approach. }\end{array}$ & $\begin{array}{l}8 \text { sessions of emotion } \\
\text { focused therapy (EFT) that } \\
\text { each session lasted } 50 \\
\text { minutes }\end{array}$ & Weekly \\
\hline Karimi (2012) & ----- & ----- & ----- & weekly \\
\hline Yousefi (2014) & counselor & $\begin{array}{l}\text { trained } \\
\text { counselor } \\
\text { in the } \\
\text { Counseling } \\
\text { Center }\end{array}$ & $\begin{array}{l}8 \text { sessions of } 1 \text { hour based } \\
\text { on Format and content } \\
\text { identified } \\
\text { for sessions }\end{array}$ & Two times a week. \\
\hline Momeni Javid (2014) & ----- & ----- & $\begin{array}{l}9 \text { sessions that each session } \\
\text { lasted } 1 \text { hour. Format and } \\
\text { content identified for } \\
\text { sessions. }\end{array}$ & per week \\
\hline
\end{tabular}

\footnotetext{
*No information
} 
Table 2. Quality of intervention* evidence

\begin{tabular}{|c|c|c|c|c|}
\hline Study & Sessions $<=2$ & $\begin{array}{l}\text { Having } \\
\text { Follow-up }\end{array}$ & $\begin{array}{lll}\begin{array}{l}\text { Intervention } \\
\text { items }\end{array} & \text { fidelity }<3 \\
\end{array}$ & Quality1 \\
\hline Hosseinian (2012) & --- & -1 & -1 & Low $(-2)$ \\
\hline Asadpour (2012) & --- & -1 & --- & $\begin{array}{l}\text { Moderate } \\
(-1)\end{array}$ \\
\hline Zarepour (2010) & --- & -1 & -1 & Low $(-2)$ \\
\hline Salimi (2012) & ---- & -1 & -1 & Low $(-2)$ \\
\hline Nasr Isfahani (2013) & --- & -1 & -1 & Low $(-2)$ \\
\hline Etemadi (2006) & --- & -1 & --- & $\begin{array}{l}\text { Moderate } \\
(-1)\end{array}$ \\
\hline Ebrahimi (2011) & --- & --- & -1 & $\begin{array}{l}\text { Moderate } \\
(-1)\end{array}$ \\
\hline Rezaei (2013) & --- & -1 & --- & $\begin{array}{l}\text { Moderate } \\
(-1)\end{array}$ \\
\hline Shakarami (2014) & --- & --- & -1 & $\begin{array}{l}\text { Moderate } \\
(-1)\end{array}$ \\
\hline Ghadam Kheir (2013) & --- & -1 & -1 & Low $(-2)$ \\
\hline Mazlomi (2012) & --- & -1 & -1 & Low $(-2)$ \\
\hline Etemadi (2014) & --- & -1 & -1 & Low (-2) \\
\hline Hosseini Zand (2013) & --- & --- & --- & High (0) \\
\hline Shariatzadeh (2014) & --- & -1 & -1 & Low $(-2)$ \\
\hline Oulia (2006) & --- & -1 & -1 & Low $(-2)$ \\
\hline Babaei Garmkhani (2014) & --- & -1 & -1 & Low $(-2)$ \\
\hline Botlani (2010) & --- & --- & -1 & $\begin{array}{l}\text { Moderate } \\
(-1)\end{array}$ \\
\hline Hosseini (2013) & --- & -1 & -1 & Low $(-2)$ \\
\hline Nasr Isfahani (2010) & --- & -1 & -1 & Low $(-2)$ \\
\hline Bahrami (2009) & --- & --- & --- & $\operatorname{High}(0)$ \\
\hline Durana (1997) & --- & --- & --- & $\operatorname{High}(0)$ \\
\hline Khanjani Veshki (2012) & --- & -1 & ---- & $\begin{array}{l}\text { Moderate } \\
(-1)\end{array}$ \\
\hline Duffey (2004) & -1 & -1 & ---- & Low $(-2)$ \\
\hline Nayari (2014) & --- & --- & -1 & $\begin{array}{l}\text { Moderate } \\
(-1)\end{array}$ \\
\hline Hajian (2013) & --- & -1 & -1 & Low $(-2)$ \\
\hline Nasirnejhad Karaj (2014) & --- & -1 & -1 & Low (-2) \\
\hline Mami (2015) & --- & -1 & --- & $\begin{array}{l}\text { Moderate } \\
(-1)\end{array}$ \\
\hline Farbod (2014) & --- & -1 & -1 & Low $(-2)$ \\
\hline Mohamadi (2013) & $\begin{array}{l}\text { No } \\
\text { information }\end{array}$ & -1 & -1 & Low $(-2)$ \\
\hline Coutta (2002) & $\begin{array}{l}\text { No } \\
\text { information }\end{array}$ & --- & --- & $\operatorname{High}(0)$ \\
\hline $\begin{array}{l}\text { Nasirnejhad } \\
\text { (2014) }\end{array}$ & --- & -1 & -1 & Low $(-2)$ \\
\hline Sharifian (2011) & --- & --- & -1 & $\begin{array}{l}\text { Moderate } \\
(-1)\end{array}$ \\
\hline Hickmon (1997) & --- & -1 & --- & $\begin{array}{l}\text { Moderate } \\
(-1)\end{array}$ \\
\hline Soltani (2013) & --- & -1 & -1 & Low $(-2)$ \\
\hline
\end{tabular}




\begin{tabular}{|c|c|c|c|c|}
\hline Study & Sessions $<=2$ & $\begin{array}{l}\text { Having } \\
\text { Follow-up }\end{array}$ & $\begin{array}{ll}\text { Intervention fidelity }<3 \\
\text { items }\end{array}$ & Quality1 \\
\hline Denton (2000) & --- & -1 & ---- & $\begin{array}{l}\text { Moderate } \\
(-1)\end{array}$ \\
\hline Karimi (2012) & --- & -1 & -1 & Low $(-2)$ \\
\hline Yousefi (2014) & --- & --- & --- & High (0) \\
\hline Momeni Javid (2014) & --- & --- & -1 & $\begin{array}{l}\text { Moderate } \\
(-1)\end{array}$ \\
\hline
\end{tabular}

*Quality of the studies downgraded for each of the following studies: 1) implementing intervention in less than two sessions, 2) lack of follow-up, 3) the accuracy of reported interventional information for fewer than three items

Table 3. Quality of evidence*

\begin{tabular}{|c|c|c|c|c|c|}
\hline Study & $\begin{array}{l}\text { Randomization; } \\
\text { allocation } \\
\text { concealment }\end{array}$ & $\begin{array}{l}\text { Losses > } \\
20 \%\end{array}$ & $\begin{array}{l}\text { Blinding Quality } \\
\text { of evidence1 }\end{array}$ & $\begin{array}{l}\text { Intervention } \\
\text { quality }\end{array}$ & $\begin{array}{l}\text { Quality of } \\
\text { evidence1 }\end{array}$ \\
\hline Hosseinian (2012) & -1 & No information & ---- & -1 & Low(-2) \\
\hline Asadpour (2012) & -1 & No information & ----- & ----- & Moderate(-1) \\
\hline Zarepour (2010) & -1 & No information & --- & -1 & Low(-2) \\
\hline Salimi (2012) & -1 & No information & --- & -1 & $\operatorname{Low}(-2)$ \\
\hline Nasr Isfahani (2013) & -1 & No information & --- & -1 & $\operatorname{Low}(-2)$ \\
\hline Etemadi (2006) & -1 & No information & --- & --- & Moderate(-1) \\
\hline Ebrahimi (2011) & -1 & No information & --- & --- & Moderate(-1) \\
\hline Rezaei (2013) & -1 & No information & --- & --- & Moderate(-1) \\
\hline Shakarami (2014) & -1 & No information & --- & --- & Moderate(-1) \\
\hline Ghadam Kheir (2013) & -1 & No information & --- & -1 & Low $(-2)$ \\
\hline Mazlomi (2012) & -1 & No information & --- & -1 & $\operatorname{Low}(-2)$ \\
\hline Etemadi (2014) & -1 & --- & --- & -1 & Low(-2) \\
\hline Hosseini Zand (2013) & -1 & No information & --- & --- & Moderate(-1) \\
\hline Shariatzadeh (2014) & -1 & No information & --- & -1 & Low(-2) \\
\hline Oulia (2006) & -1 & --- & --- & -1 & Low(-2) \\
\hline $\begin{array}{l}\text { Babaei } \quad \text { Garmkhani } \\
\text { (2014) }\end{array}$ & -1 & No information & --- & -1 & $\operatorname{Low}(-2)$ \\
\hline Botlani (2010) & -1 & No information & --- & --- & Moderate(-1) \\
\hline Hosseini (2013) & -1 & No information & --- & -1 & $\operatorname{Low}(-2)$ \\
\hline Nasr Isfahani (2010) & -1 & No information & --- & -1 & $\operatorname{Low}(-2)$ \\
\hline Bahrami (2005) & -1 & No information & --- & --- & Moderate(-1) \\
\hline Durana (1997) & -1 & --- - & --- & --- & Moderate(-1) \\
\hline $\begin{array}{l}\text { Khanjani } \quad \text { Veshki } \\
(2012)\end{array}$ & -1 & No information & --- & --- & Moderate(-1) \\
\hline Duffey (2004) & -1 & No information & --- & -1 & Low(-2) \\
\hline Nayari (2014) & -1 & No information & --- & --- & Moderate(-1) \\
\hline Hajian (2013) & -1 & No information & --- & -1 & Low(-2) \\
\hline $\begin{array}{l}\text { Nasirnejhad Karaj } \\
\text { (2014) }\end{array}$ & -1 & No information & --- & -1 & $\operatorname{Low}(-2)$ \\
\hline Mami (2015) & -1 & No information & --- & --- & Moderate(-1) \\
\hline Farbod (2014) & -1 & No information & --- & -1 & Low(-2) \\
\hline Mohamadi (2013) & -1 & No information & --- & -1 & $\operatorname{Low}(-2)$ \\
\hline Coutta (2002) & -1 & -1 & --- & --- & Low(-2) \\
\hline
\end{tabular}




\begin{tabular}{|c|c|c|c|c|c|}
\hline Study & $\begin{array}{l}\text { Randomization; } \\
\text { allocation } \\
\text { concealment }\end{array}$ & $\begin{array}{l}\text { Losses > } \\
20 \%\end{array}$ & $\begin{array}{l}\text { Blinding Quality } \\
\text { of evidence } 1\end{array}$ & $\begin{array}{l}\text { Intervention } \\
\text { quality }\end{array}$ & $\begin{array}{l}\text { Quality of } \\
\text { evidence1 }\end{array}$ \\
\hline $\begin{array}{ll}\text { Nasirnejhad } & \text { Tehran } \\
\text { (2014) } & \end{array}$ & -1 & No information & --- & -1 & $\operatorname{Low}(-2)$ \\
\hline Sharifian (2011) & -1 & No information & --- & --- & Moderate(-1) \\
\hline Hickmon (1997) & -1 & No information & +1 & --- & $\operatorname{High}(0)$ \\
\hline Soltani (2013) & -1 & No information & --- & -1 & $\operatorname{Low}(-2)$ \\
\hline Denton (2000) & -1 & -1 & --- & --- & $\operatorname{Low}(-2)$ \\
\hline Karimi (2012) & -1 & No information & --- & -1 & Low(-2) \\
\hline Yousefi (2014) & -1 & No information & --- & --- & Moderate(-1) \\
\hline Momeni Javid (2014) & -1 & No information & --- & --- & Moderate(-1) \\
\hline
\end{tabular}

*Quality could be high, moderate, low, or very low. We considered these RCTs to be high quality then downgraded a level for each of the following: A) lack of information on random sequence, allocation concealed, or lack of allocation concealed B) low quality interventions (Table 2), c) loss of more than $20 \%$ at follow-up. We upgraded one level for the studies that performed some blinding.

Table 4. Characteristics of excluded studies

\begin{tabular}{ll}
\hline Study & Reason for exclusion \\
\hline Amber (2011) & Trial focused on couples with cancer \\
Leclerc & Trial focused on young adults with first psychological episode \\
Chambers (2014) & Trial focused on men with localised prostate cancer and their female partners \\
Zarei (2014) & $\begin{array}{l}\text { Trial focused on spouses of war-disabled affiliated with markers and self } \\
\text { sacrifices }\end{array}$ \\
Jun (2011) & Trial focused on Breast Cancer Survivors \\
Heather (2013) & Trial focused on men with localised prostate cancer \\
Robertson (2014) & Trial focused on Patients with prostate cancer and their partners \\
Reese (2012) & Trial focused on couple who had facing colorectal cancer. \\
Reese (2014) & Trial focused on couple who had facing colorectal cancer. \\
Julia (2009) & Trial focused on Patients with breast cancer \\
Jung (2005) & Trial focused on male patients with spinal cord injuries \\
Kerri (2012) & Trial focused on prostate cancer survivors (PCS) and their spouses \\
Manne (2004) & Trial focused on women with breast cancer and their partners \\
Nho (2013) & Trial focused on Women with Gynecologic Cancer and Their Husbands \\
Otto (2015) & Trial focused on women with breast cancer and their intimate partners \\
Gol (2013) & Trial focused on depressed patients \\
DeMarco (2009) & Trial focused on women living with or at risk for HIV. \\
Manne (2011) & Trial focused on Men Diagnosed with Prostate Cancer and Their Partners \\
Hummel (2015) & Trial focused on breast cancer survivors \\
Sidddons (2013) & Trial focused on men with localised prostate cancer. \\
Edward (1995) & Trial focused on depressed married women \\
Babapour & Kheiroddin \\
(2012) & Trial focused on chemical patient couples \\
Hamedi (2011) & Trial focused on addicted man and their Wives. \\
Sadrejahani (2009) & Trial focused on addicts and their wives \\
Kazemian (2013) & Trial focused on infertile Couples \\
\hline
\end{tabular}


Table 5. The intimacy-enhancing interventions in different dimensions

\begin{tabular}{|c|c|c|}
\hline Dimension & Intervention & Authors \\
\hline \multirow{10}{*}{ Emotional intimacy } & Communication skill & Hosseinian (2012), Mazlomi (2012) \\
\hline & Relationship Therapy & Etemadi (2014) \\
\hline & Relationship enhancement program & Ebrahimi (2011) \\
\hline & Marital enrichment & Oulia (2006), Bahrami (2009) \\
\hline & Solution-focused couples therapy & Hajian (2013) \\
\hline & Solution-Focused Group Counseling & Hosseini (2013) \\
\hline & Cognitive-behaviour couple therapy & Etemadi (2006) \\
\hline & Training of Islamic Lifestyle & Rezaei (2013) \\
\hline & Emotional focused couple therapy & Soltani (2013), Asadpour (2012) \\
\hline & (narrative therapy) & Mohamadi (2013) \\
\hline \multirow{10}{*}{ Psychological intimacy } & Communication skill & Hosseinian (2012), Mazlomi (2012) \\
\hline & Relationship enhancement program & Ebrahimi (2011) \\
\hline & Marital enrichment & Oulia (2006), Bahrami (2009) \\
\hline & Solution-focused couples therapy & Hajian (2013) \\
\hline & Solution-Focused Group Counseling & Hosseini (2013) \\
\hline & Cognitive-behavior couple therapy & Etemadi (2006) \\
\hline & Training of Islamic Lifestyle & Rezaei (2013) \\
\hline & Emotional focused couple therapy & Soltani (2013), Asadpour (2012) \\
\hline & Problem Solving Training & Zarepour (2010) \\
\hline & Communication skill & Hosseinian (2012), Mazlomi (2012) \\
\hline \multirow{6}{*}{ Physical intimacy } & Relationship enhancement program & Ebrahimi (2011) \\
\hline & Relationship Therapy & Etemadi (2014) \\
\hline & Problem Solving Training & Zarepour (2010) \\
\hline & Solution-focused couples therapy & Hajian (2013) \\
\hline & Training of Islamic Lifestyle & Rezaei (2013) \\
\hline & Emotional focused couple therapy & Soltani (2013), Asadpour (2012) \\
\hline \multirow{11}{*}{ Sexual intimacy } & Communication skill & Hosseinian (2012), Mazlomi (2012 \\
\hline & Relationship enhancement program & Ebrahimi (2011) \\
\hline & Solution-focused couples therapy & Hajian (2013) \\
\hline & Solution-Focused Group Counseling & Hosseini (2013) \\
\hline & Cognitive-behavior couple therapy & Etemadi (2006) \\
\hline & Training of Islamic Lifestyle & Rezaei (2013) \\
\hline & Islamic couple therapy & Hosseini Zand (2013) \\
\hline & Sex education & $\begin{array}{l}\text { Shakarami (2014), Salimi (2012), Khanjani } \\
\text { veshki (2012) }\end{array}$ \\
\hline & Attachment-based couple therapy & Botlani (2010) \\
\hline & Emotional focused couple therapy & Soltani (2013), Asadpour (2012) \\
\hline & Training Positive Thinking & Nasiri Nejad (2014) \\
\hline \multirow{3}{*}{ Temporal intimacy } & Emotional focused couple therapy & Soltani (2013) \\
\hline & Communication skill & Mazlomi (2012) \\
\hline & Solution-Focused Group Counseling & Hosseini (2013) \\
\hline \multirow{5}{*}{$\begin{array}{l}\text { Communicational } \\
\text { intimacy }\end{array}$} & Relationship enhancement program & Ebrahimi (2011) \\
\hline & Solution-focused couples therapy & Hajian (2013) \\
\hline & Marital enrichment & Oulia (2006), Bahrami (2009) \\
\hline & Training of Islamic Lifestyle & Rezaei (2013) \\
\hline & Emotional focused couple therapy & Soltani (2013), Asadpour (2012) \\
\hline
\end{tabular}




\begin{tabular}{|c|c|c|}
\hline Dimension & Intervention & Authors \\
\hline \multirow{6}{*}{$\begin{array}{l}\text { Social-Recreational } \\
\text { intimacy }\end{array}$} & (narrative therapy) & Mohamadi (2013) \\
\hline & Communication skill & Hosseinian (2012), Mazlomi (2012) \\
\hline & Relationship enhancement program & Ebrahimi (2011) \\
\hline & Marital enrichment & Oulia (2006), Bahrami (2009) \\
\hline & Emotionally focused couple therapy & Asadpour (2012) \\
\hline & Training of Islamic Lifestyle & Rezaei (2013) \\
\hline \multirow[t]{3}{*}{ Aesthetic intimacy } & Communication skill & Mazlomi (2012) \\
\hline & Communication skill & Hosseinian (2012), Mazlomi (2012) \\
\hline & Relationship enhancement program & Ebrahimi (2011) \\
\hline \multirow[t]{5}{*}{ Spiritual intimacy } & Marital enrichment & Oulia (2006), Bahrami (2009) \\
\hline & Emotionally focused couple therapy & Asadpour (2012) \\
\hline & Training of Islamic Lifestyle & Rezaei (2013) \\
\hline & Communication skill & Hosseinian (2012), Mazlomi (2012) \\
\hline & Relationship enhancement program & Ebrahimi (2011) \\
\hline \multirow{11}{*}{ Intellectual intimacy } & Marital enrichment & Oulia (2006), Bahrami (2009) \\
\hline & Emotional focused couple therapy & Soltani (2013), Asadpour (2012) \\
\hline & Problem Solving Training & Zarepour (2010) \\
\hline & Solution-focused couples therapy & Hajian (2013) \\
\hline & Communication skill & $\begin{array}{l}\text { Farbod (2014), Sharifian (2011), Karim I } \\
\text { (2012) }\end{array}$ \\
\hline & Problem Solving Training & Zarepour (2010) \\
\hline & $\begin{array}{l}\text { Training solution-focused couples } \\
\text { therapy }\end{array}$ & Hosseini (2013) \\
\hline & Dream sharing & Duffey (2004) \\
\hline & Marital enrichment & Coutta(2002), Hickmon (1997) \\
\hline & Training Positive Thinking & Nasirnejhad (2014) \\
\hline & Cognitive- behavior couple therapy & $\begin{array}{l}\text { Mami (2015), Etemadi } \\
\text { BabaeiGarmkhani (2014) }\end{array}$ \\
\hline \multirow[t]{8}{*}{ Total intimacy } & Meaning-centered training & Nasr Isfahani (2013) \\
\hline & Choice theory training & Nasr Isfahani (2010) \\
\hline & $\begin{array}{l}\text { Rational - emotional behavioral } \\
\text { therapy }\end{array}$ & Ghadam kheir (2013) \\
\hline & Foot massage & Uhm (2010) \\
\hline & $\begin{array}{l}\text { Rogers Self Theory and Ellis } \\
\text { Rational Theory }\end{array}$ & Yousefi (2014) \\
\hline & $\begin{array}{l}\text { Group training of transactional } \\
\text { analysis }\end{array}$ & Nayeri (2014) \\
\hline & Enhancing marital intimacy & Durana (1997) \\
\hline & Emotion focused therapy & Denton (2000) \\
\hline
\end{tabular}

\section{Search Results}

Sixty six sources were provided by the search from 1995 to April 2015. After reviewing the various titles and abstracts, 25 studies were excluded from review due to the lack of consideration of study criteria (the 25 studies that were excluded from review included 9 studies in the USA, 6 from Iran, 2 from Canada, 3 in each of Australia and Korea, whereas 1 was completed in each of England and the Netherlands, although they focusing on respondents with drug abuse and chronic health conditions) (Table 4). Finally, 39 trials met the inclusion criteria (Figure 1). The total number of participants was 1981 people, and the number of participants in each study was from 24 to 216 people. Average number of participants in each trial was 50.79 people. Thirty three studies were conducted in Iran, and the six others were conducted in America and Korea. Sixteen trials focused 
on women, 20 trials focused on couples, and 3 trials focused on men and women. Studies varied in provided educating content and format.

Eleven interventions had follow-up (Bahrami, Oulia, \& Isanezhad, 2009; Botlani, Ahmadi, Bahrami, Shahsiah, \& Mohebbi, 2010; Coutta, 2001; Durana, 1997; Ebrahimi, Sanaei Zaker, \& Nazari, 2011; Hosseini Zand et al., 2013; Momeni Javid, Soveyzi, \& Mousavi, 2014; Nayeri et al., 2014; Shakarami, Davarniya, Zahrakar, \& Gohari, 2014; Sharifian, Najafi, \& Shaghaghi, 2011; Yousefi \& Kiani, 2014) and 28 interventions lacked follow-up. Time of interventions was from one 4-hour workshop (Duffey) to 120-hour interventions for 4 to 5 months (Durana).

The quality of interventions was high in five studies, moderate in 13 studies was, and low in 20 studies. However, due to the limitations in the language in one study (the full text of the article was in Korean), it was not feasible to ensure the quality of the intervention (Table 2). The quality evidence was low for 22 interventions, moderate for 15 interventions, and high for one intervention (Table 3). Findings from studies were categorized in 11 categories as the intimacy promoting interventions in dimensions of emotional, psychological, physical, sexual, temporal, communicational, social and recreational, aesthetic, spiritual, intellectual intimacy, overall dimension, and total intimacy and are shown in Table 5.

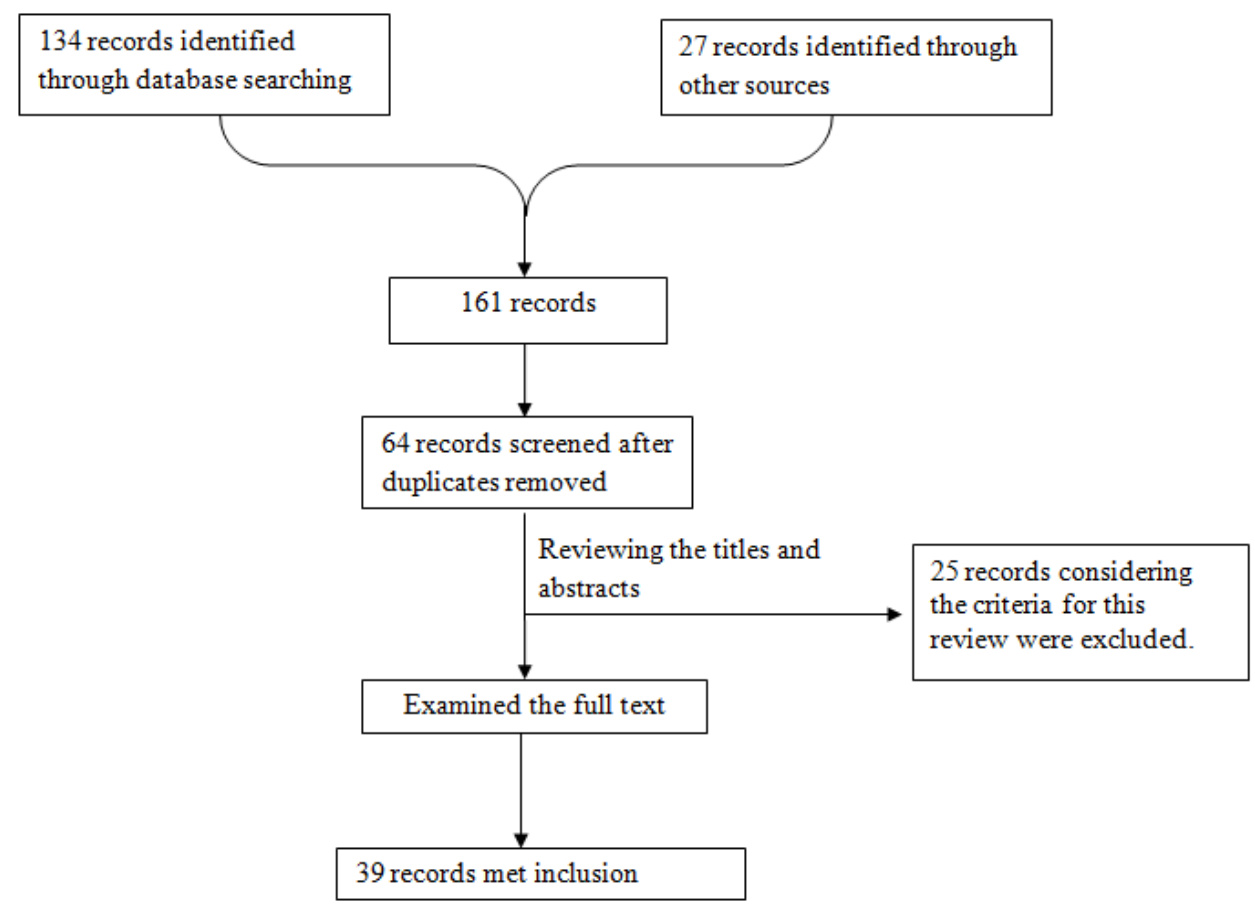

Figure 1. Result of the search

\section{Discussion}

\subsection{Emotional Intimacy}

emotional intimacy has been described as to share all the emotions, both positive and negative feelings with the spouse (Bagarozzi, 2001). Studies show that training and enriching the communication skills and communication therapy can contribute to the promotion of emotional intimacy (Ebrahimi et al., 2011; Etimadi, Jafari, \& Seyah, 2014; Hosseinian, Yazdi, \& Tabatabaei, 2012; Mazlomi, Dolatshahi, \& Nazari, 2012). In these studies, participants were trained in some of the most important skills including conflict resolution by understanding the hidden needs and feelings of the spouse, understanding how to ask the needs and expectations, Identification of the impact of incorrect beliefs and expectations of spouses on the creation of conflicts and reduction of intimacy and active listening (Mazlomi et al., 2012), increasing self-awareness, knowing the spouse, getting familiar with each other's needs and losses, renewing the memories of the past and improving the relations (Etimadi et al., 2014) and training communication skills based on Miller's theory. So that in this plan, women were instructed the skills of speaking so as to convey information to the spouse, skills of listening, and skills of problem solving and planning in order to solve problems and identify effective communication styles (Hosseinian et al., 2012). 
Solution-focused training plays an important role in increasing this dimension of intimacy. In this study, the couples were trained in six 90-minute training sessions to improve relationships and communication, evaluate the level of marital conflict and the nature of the problem, detect the chief complaint and define the problem, set a goal, examine solutions, formulate circles to find the solutions of the problems, and give the old and common solutions using intensive courses of couple therapy along with doing some homework in each session (Hajian \& Mohammadi, 2013). The study showed that group counseling in the solution-focused method enhances the emotional intimacy (Hosseini, Majd, \& GHamari, 2013). The other studies also stated that emotion-focused couple therapy can promote this dimension of intimacy (Asadpour, Nazari, Zaker, \& Shaghaghi, 2012; Soltani et al., 2013). The 9-stage emotion-focused therapy of couples consists of description of the issues related to the conflict, identification of negative interaction circle that causes distress in couples, access unexplored emotions that are based on interactive conditions, formulation of the problem baced on emotion, like anger, disgust, fear, happiness, sadness and surprise. and attachment-focused needs, increase of the understanding of self emotions and personal needs that have been ignored, increase of accepting experiences of each spouse by the other party, creation of new ways of communicating, facilitation of expressing emotional needs and demands, facilitation of the development of new solutions for old problems and finally integration and reinforcement of new situations (Asadpour et al., 2012; Soltani et al., 2013).

Etemadi et al. (2006) showed in their study that the use of cognitive-behavioral techniques can promote emotional intimacy. In the cognitive behavioral techniques, participants were studied in terms of having unrealistic expectations and beliefs about intimacy and sexual relationships and the destructive effects of such behaviors on feelings, eliminating misunderstandings arising from misconceptions or different understanding, assessing the problems associated with the message sender and receiver and training communication skills, creating empathic understanding and active listening comprehension skills, training problem solving skills, and exploring the conflicts between spouses weekly and along with assignments (Etemadi, Navvabi Nezhad, Ahmadi, \& Farzad, 2006). Other interventions to promote emotional intimacy can be pointed out as narrative therapy (Mohammadi, Sohrabi, \& Aghdam, 2013), the Islamic lifestyle (Rezaei et al., 2013), and enriching the marital life (Bahrami et al., 2009; Oulia et al., 2006).

\subsection{Psychological Intimacy}

Psychological intimacy involves sharing personal issues, information, hopes, fears, desires, and feelings about the self with a spouse (Bagarozzi, 2001). The study of Ebrahimi et al. (2011) showed that enriching communication plays an important role in enhancing this dimension of intimacy. Here, in the relationship enrichment program, expressive skills, empathic listening, correct simultaneous way of speaking and listening and comparing it with the non-skilled dialogue, conflict resolution skill, self -change skill, and the skill of helping the spouse to change of the participants of the study were investigated (Ebrahimi et al., 2011). The studies of Hosseinian et al. (2012) and Mazlomi et al. (2012) came across results in line with the study of Ebrahimi (2011) and showed that enriching communication skills contributes to a rise in intimacy. Education of problem-solving skill among couples leads to increasing psychological intimacy (Zarepour, 2010), that is in line with the study of Hajian (2013) (Hajian \& Mohammadi, 2013). Zarepour performed the education of problem-solving skill in order to take a positive and optimistic attitude towards the problem and the ability of the couples to deal with it, identify problems and obstacles to solve the problem, identify realistic objectives agreed by the couples, evaluate each solution and select the best solution, and implement the selected solution in the real life (Zarepour, 2010). Other interventions that can promote the psychological intimacy can be emotion focused couple therapy skill (Asadpour et al., 2012; Soltani et al., 2013) and the Islamic lifestyle approach. The Islamic life style approach is based on Islamic rules and principles more expounded on in the following sections. (Rezaei et al., 2013)

\subsection{Physical Intimacy}

Physical intimacy is the partner's need to physical contact such as hugging, holding hands and non-sexual touch (Bagarozzi, 2001). Education of problem-solving skill is effective in increasing this dimension of intimacy (Zarepour, 2010). The studies of Hajian (2013) and Hosseini (2013) were consistent with the study of Zarepour (2010) and showed that solution-focused training leads to increasing physical intimacy (Hajian \& Mohammadi, 2013; Hosseini et al., 2013). Emotion-focused couple therapy can promote the physical intimacy of the couples (Asadpour et al., 2012). The study of Soltani (2013) is also in line with the study of Asadpour (Soltani et al., 2013). Other interventions that can promote the physical intimacy can be communication enriching (Ebrahimi et al., 2011; Etimadi et al., 2014; Hosseinian et al., 2012; Mazlomi et al., 2012) and Islamic lifestyle training (Rezaei et al., 2013). 


\subsection{Sexual Intimacy}

Sexual intimacy involves the expression of thoughts, feelings, and desires that have sexual nature and are planned to arouse sexual stimulation and sexual satisfaction (Bagarozzi, 2001). One of the ways to increase sexual intimacy is to present sex education to couples (Shakarami et al., 2014). Education and counseling in sexual dimension cause the couples to get enough awareness in this field and take effective steps to deal with their sexual problems and promote sexual intimacy (Zand et al., 2013). Sex education based on cognitive-behavioral techniques is effective to improve sexual intimacy (Veshki et al., 2012). Sex education increases sexual intimacy (Salimi \& Fatehizadeh, 2012; Shakarami et al., 2014). In sex education, participants become familiar with physiology and sexual behavior and also receive education on topics such as modifying the myths about sexual matters, shaping the sexual intimacy and appropriate sexual techniques, and getting familiar with some of the most common sexual disorders (Shakarami et al., 2014). In addition, in the study of Salimi (2012), participants received trainings such as relaxation and fantasy skill, attention and awareness of the Sensory symptoms, expression of emotion and self -sexual expression, establishment of sexual intimacy, increase of positive self-talk, communication skill, increase of positive interactions, and problem solving (Salimi \& Fatehizadeh, 2012).

Couples' communication skill improvement increased sexual intimacy (Mazlomi et al., 2012). The study of Hosseinian (2012) and Ebrahimi (2011) are in line with the study of Mazlomi (2012) (Ebrahimi et al., 2011; Hosseinian et al., 2012). While Etemadi (2014) showed that communication therapy does not improve the sexual intimacy (Etimadi et al., 2014). Nasiri Nejad (2014) found that educating positive thinking is conducive to sexual function and sexual intimacy of the spouses. In this study, participants were first familiarized with the need to positive thinking, different coping styles, and the ways to forming thinking and attitudes. The participants were then familiarized with their negative thoughts and modification techniques and also with positive thinking and its effect on the life based on cognitive-behavioral technique. Applying the ABC theory of, (The ABC Model A major aid in cognitive therapy is what Albert Ellis (1957) called the ABC Technique of Irrational Beliefs. The first three steps analyze the process by which a person has developed irrational beliefs including: A - Activating Event or objective situation, B - Beliefs and C - Consequence. Ellis believes that it is not the activating event (A) that causes negative emotional and behavioral consequences $(C)$. Rather, a person interprets these events unrealistically and, therefore, has an irrational belief system (B) that helps cause the consequences (C) (McLeod, 2008)). The participants were trained in forming positive thoughts for example: 1) Im responsible and in control of my life. 2) Circumstances are what they are, but I can choose my attitude towards them. And 3) Every challenge that comes along is an opportunity to learn and grow. 4) I am getting better every day.). Training techniques to stop negative thoughts, boosting self confidence, and adding laughter and sports to life were other positive thinking techniques which were relied upon (Nejad, Nazari, \& Bahrainian, 2014) .Other interventions to promote sexual intimacy can be solution-focused training (Hajian \& Mohammadi, 2013; Hosseini et al., 2013), use of cognitive-behavioral techniques (Etemadi et al., 2006), the Islamic lifestyle (Rezaei et al., 2013), couple therapy (Zand et al., 2013), couple therapy based on attachment (Botlani et al., 2010) and emotion-focused couple therapy (Asadpour et al., 2012; Soltani et al., 2013).

\subsection{Temporal Intimacy}

Temporal intimacy indicates the extent to which couples tend to spend their daily time with their spouses on intimate activities (Bagarozzi, 2001). The study of Soltani et al. (2013) showed that emotion-focused couple therapy can promote this dimension of (Soltani et al., 2013). The first hypotheses on excitement-based treatments contend that the most effective factor in creating and maintaining marital intimacy is the type of the existing chain of excitement. Johnson (2004) predicts that excitement-based treatment (emphasizing sympathy, self-expression, deep understanding of one's self needs and the partner's needs, acceptance, expression of ideas and feelings and creation of an emotional environment, all of which are considered as essential elements in an intimate relationship) can play a powerful role in increasing intimacy in couples (Hamedi, Abadi, Navabinejad, \& Delavar, 2013). Other interventions that can be pointed out to increase intimacy are communication skill training (Mazlomi et al., 2012) and solution-focused group counseling, in Solution-based treatment focuses on the activities both of the spouses enjoy and encourages them to do those activities again. Recommending the couple to walk and have recreation together without the presence children can be significant help to them in an optimal use of their time (Hosseini et al., 2013). 


\subsection{Communication Intimacy}

Communication intimacy is defined as the creation of a relationship with respect, commitment, and positive emotions in such a way that the spouses feel valued and respected in this communication (Oulia et al., 2006). Bahrami (2009) carried out a study with the purpose of enriching marital life, and the intervention group was trained to have intimacy, improve sex issues, manage household, restructure cognition, and learn conflict resolution skill. The results showed that training marital life enrichment enhances communication intimacy (Bahrami et al., 2009). The results of the study of Oulia (2006) are consistent with the above study (Oulia et al., 2006). Hajian (2013) reported that group solution-focused training is related to promoting communication intimacy by promoting intimacy (Hajian \& Mohammadi, 2013). Other study also showed that communication enrichment is associated with increased communication intimacy (Ebrahimi et al., 2011; Hosseinian et al., 2012).

Moreover, Soltani (2013) stated that the emotion-focused therapy increased communication intimacy (Soltani et al., 2013) that the study of Asadpour (2012) is consistent with the above study (Asadpour et al., 2012). Other interventions promoting communication intimacy include narrative therapy (Mohammadi et al., 2013) and Islamic lifestyle (Rezaei et al., 2013).

\subsection{Social-Recreational Intimacy}

Social recreational intimacy requires involving the spouse in responsibilities, passing holidays, enjoyable activities and leisure time, and expressing experiences and daily events (Bagarozzi, 2001). It was shown in the studies that communication skill training increases this dimension of intimacy (Ebrahimi et al., 2011; Hosseinian et al., 2012; Mazlomi et al., 2012); however, Etemadi (2014) showed that communication therapy has no positive effect in promoting social recreational intimacy (Etimadi et al., 2014). Asadpour (2012) demonstrated that emotion-focused couple therapy can promote social recreational intimacy while Soltani (2013) stated that emotion-focused couple therapy has no significant effect on increasing this type of intimacy (Asadpour et al., 2012; Soltani et al., 2013). In emotion-focused couple therapy, the first hypotheses on excitement-based treatments contend that the most effective factor in creating and maintaining marital intimacy is the type of the existing chain of excitement. Johnson (2004) predicts that excitement-based treatment (emphasizing sympathy, self-expression, deep understanding of one's self needs and the partner's needs, acceptance, expression of ideas and feelings and creation of an emotional environment, all of which are considered as essential elements in an intimate relationship) can play a powerful role in increasing intimacy in couples (Hamedi et al., 2013). Rezaei (2013) showed in his study that the Islamic lifestyle training increases recreational intimacy between spouses and that a summary of Islamic lifestyle training content includes the definition of marital intimacy, expression of couples' expectations from their marital life, Islam's idea about intimacy and the ways to increase it, verbal and nonverbal communications of the spouses, role of forgiveness in the conjugal life, guidance in order to enhance the relationships among couples, rights of spouses towards each other and respecting the boundaries in the families, sex customs in Islam and respect for privacy in sexual relationships, procedures of creating peace in the family, and methods of conflict resolution in the family (Rezaei et al., 2013).

\subsection{Aesthetic Intimacy}

Aesthetic intimacy needs sharing feelings, thoughts and beliefs that are Beautiful exciting in one's opinion (Aesthetic intimacy needs sharing feelings, thoughts, and beliefs which are beautiful excitements in one's opinion, such as wonders of nature and the cosmos, music, art, poetry, etc. (Bagarozzi, 2001). Mazlomi (2012) demonstrated in that communication skill training can promote the aesthetic intimacy (Mazlomi et al., 2012), while the study of Etemadi (2006), which was performed to evaluate the effect of cognitive behavioral techniques training on intimacy and the intervention group, was trained skills of communication, problem solving, and conflict. Besides, cognitive factors showed that the above skills do not have any effects on improving this dimension of intimacy (Etemadi et al., 2006).

\subsection{Spiritual Intimacy}

Religious intimacy is described as to express your thoughts, feelings, beliefs and experiences about religion, supernatural issues, moral values, life after death, and the relationship with God for your spouse (Bagarozzi, 2001). Mazlomi et al. (2012) showed that promoting communication skill of couples increases the intimacy in this dimension (Mazlomi et al., 2012). The other studies are in line with the study of Mazlomi (2012) (Ebrahimi et al., 2011; Hosseinian et al., 2012), while Etemadi et al. (2014) reported that communication therapy does not improve religious intimacy (Etimadi et al., 2014). Asadpour (2012) also showed that emotion-focused couple therapy can promote religious intimacy; on the other hand Soltani (2013) showed that emotion-focused couple therapy has no significant effect on increasing this dimension of intimacy (Asadpour et al., 2012; Soltani et al., 2013). Also Oulia (2006) reported that the marital life enrichment can promote religious intimacy that is in line 
with the study of Bahrami (2009) (Bahrami et al., 2009; Oulia et al., 2006). The results of the study of Rezaei et al. (2013) also showed that religious intimacy is effective in improving the Islamic lifestyle (Rezaei et al., 2013).

\subsection{Intellectual Intimacy}

Intellectual intimacy is the need to transfer and restate important thoughts and beliefs with the spouse (Bagarozzi, 2001). The study showed that enrichment of marital life can promote the intellectual intimacy (Oulia et al., 2006). The other study is in line with the above study (Bahrami et al., 2009). The results of the study of Mazlomi et al. (2012) stated that improving the communication skill of couples increases intellectual intimacy (Mazlomi et al., 2012). The other studies are in line with the study of Mazlomi (2012) (Ebrahimi et al., 2011; Hosseinian et al., 2012), while Etemadi et al. (2014) showed that the communication therapy has no effect on the promotion of intellectual intimacy (Etimadi et al., 2014). Zarepour (2010) showed that training problem-solving skill leads to the improvement of intellectual intimacy between the couples (Zarepour, 2010). Solution-focused training of the couples can promote this aspect of intimacy (Hajian \& Mohammadi, 2013). Emotion focused therapy increases this dimension of intimacy (Soltani et al., 2013), which is in line with the study of Asadpour et al. (2012).

\subsection{Total Intimacy}

Momeni Javid et al. (2014) reported that training marital life promoting skills has an effective role to improve marital intimacy (Javid et al., 2014). The results of the other studies are in line with this study (Farbod et al., 2014; Karimi, Hasani, Soltani, Dalvand, \& Zohdi, 2012; Sharifian et al., 2011). Moreover, Zarepour (2010) showed that promoting problem-solving skill in couples is associated with increasing the overall intimacy that the study of Hosseini (2013) is in line with it (Hosseini et al., 2013; Zarepour, 2010). Sharing dreams and events can promote total intimacy (Duffey et al., 2004). Marital life enrichment of couples increases the intimacy that is in line with the study of Hickmon (1997) (Coutta, 2001; Hickmon Jr, Protinsky, \& Singh, 1997). Enrichment of intimacy promoting program increases marital intimacy (Durana, 1997). The study reported that the use of cognitive-behavioral techniques enhances the intimacy of the couples which is consistent with the other studies (Babaei Garmkhani, Madani, \& Lavasani, 2014; Etemadi et al., 2006; Mami, Roohandeh, \& Kahareh, 2015). Emotion focused therapy can promote intimacy (Denton, Burleson, Clark, Rodriguez, \& Hobbs, 2000) and emotional intellectual behavioral therapy can promote intimacy (Ghadam kheir, Ghamari Givi, Niloofar, \& Sepehri Shamlo, 2013). Education of choice theory concepts increases marital intimacy (Nasr Isfahani, 2010), while the results of the study of Shariatzadeh (2014) suggested that the effect of training choice theory in group method was not significant to increase marital intimacy (Shariatzadeh, Tabrizi, \& Ahghar, 2014). Other intimacy promoting interventions include positive thinking (Nasiri Nejad, Tork, Zahedi Rad, Nazari, \& Korivand, 2014), meaning focused training (N. Nasr Isfahani, Etemadi, \& Shafie Abadi, 2013) foot massage (Uhm, 2010), Rogers and Ellis psychotherapy (Yousefi \& Kiani, 2014) and group training of transactional analysis (Nayeri et al., 2014).

\section{Conclusion}

Overall, it can be stated from reviews that since intimacy involves the exchange of deep feelings and personal and private thoughts, promoting communication skill can play an important role in promoting intimacy in couples. In addition, according to the point that problem solving skill helps couples to evaluate the solutions to their problems and find more sense of cooperation and empathy, it could be accounted for as one of the most important factors for increasing the agreement and intimacy in couples. The depth of intimacy that people understand in their communications depends on their ability to handle correct, effective, and clear communications with the expression of feelings, needs, and desires.

Based on the results, it can be expressed that self-disclosure and empathic response can also increase intimacy because; when people trust each other and share their thoughts, feelings, and internal reality, it helps them strengthen the intimate communication in couples. Also, it can be concluded from studies that sex education and counseling helps the couples gain sufficient knowledge in this area and take effective steps to deal with sexual problems and enhance their intimacy. Generally, by promoting communication, problem solving, self-disclosure, empathic response skills, and sexual education and counseling in the form of cognitive-behavioral techniques, based on religious and cultural context of each society, an effective step can be taken to enhance marital intimacy and strengthen family bonds and stability. Therefore, it is recommended to provide and present counseling training packages to increase marital intimacy tailored to the cultural context of the society.

\subsection{Implications for Practice}

The majority of the interventions that promoted marital intimacy were quasi experimental. Interventions need to be adapted to other environments and tested again. Health care providers should consider which interventions are 
appropriate to the couple characteristics and their relationships and then use them.

\subsection{Application in Research}

The quality of many of the interventions was low and medium and did not have enough follow-up. The researchers need to design high-quality clinical trials with long-term follow-up period appropriate to the setting and resources. Also the processes of randomization and concealments are applied in designing interventions. It is also recommended that researchers measure effectiveness of interventions in raising marital intimacy.

\section{Acknowledgements}

The current review is a part of MSc. Degree in Midwifery Counseling, Maryam Kardan-Souraki. This project was mainly funded and supported by Mazandaran University of Medical Sciences, Grant No212. We appreciate Student Research Committee, Mazandaran University of Medical Sciences, Sari, Iran for supporting narrative review protocol section.

\section{Conflict of Interest}

The authors declare that there is no conflict of interests regarding the publication of this paper.

\section{References}

Asadpour, I., Nazari, A., Sanai Zaker, B., \& Shaghaghi, S. (2012). An investigation of effectiveness of the emotionally focused couple therapy on increasing marital intimacy in couples entered at counseling centers in Tehran. Research in Clinical Psychology and Counseling, 2(1), 25-38.

Bagarozzi, D. A. (2001). Enhancing intimacy in marriage: A clinician's guide. Routledge.

Bahrami, F., Oulia, N., \& Isanezhad, O. (2009). The study of the effectiveness of Enrichment Program Training (EPT) on increasing of marital satisfaction and marital intimacy. International journal of psychology, 3(1/2), $1-21$.

Boden, J. S., Fischer, J. L., \& Niehuis, S. (2010). Predicting marital adjustment from young adults' initial levels and changes in emotional intimacy over time: A 25-year longitudinal study. Journal of Adult Development, 17(3), 121-134. http://dx.doi.org/10.1007/s10804-009-9078-7

Botlani, S., Ahmadi, A., Bahrami, F., Shahsiah, M., \& Mohebbi, S. (2010). Effect of attachment-based couple therapy on sexual satisfaction and intimacy. Journal of Fundamentals of Mental Health, 12(2), 496-505.

Coutta, E. R. (2001). An investigation into the significance of an acceptance-based marital enrichment group on couple intimacy and acceptance. Available from ProQuest Dissertation \& Theses: Full Text.

Dandurand, C., \& Lafontaine, M. F. (2013). Intimacy and couple satisfaction: The moderating role of romantic attachment. International Journal of Psychological Studies, 5(1), 74-90. http://dx.doi.org/10.5539/ijps. v5n1p74

Denton, W. H., Burleson, B. R., Clark, T. E., Rodriguez, C. P., \& Hobbs, B. V. (2000). A randomized trial of emotion focused therapy for couples in a training clinic. Journal of Marital and Family Therapy, 26(1), 65-78. http://dx.doi.org/10.1111/j.1752-0606.2000.tb00277.x

Dildar, S., Sitwat, A., \& Yasin, S. (2013). Intimate enemies: Marital conflicts and conflict resolution styles in dissatisfied married couples. Middle-East Journal of Scientific Research, 15(10), 1433-1439.

Duffey, T. H., Wooten, H. R., Lumadue, C. A., \& Comstock, D. C. (2004). The effects of dream sharing on marital intimacy and satisfaction. Journal of Couple \& Relationship Therapy, 3(1), 53-68. http://dx.doi.org/ 10.1300/J398v03n01_04

Durana, C. (1997). Enhancing marital intimacy through psychoeducation: The PAIRS program. The family journal, 5(3), 204-215. http://dx.doi.org/10.1177/1066480797053004

Ebrahimi, P., Sanaei Zaker, B., \& Nazari, A. M. (2011). Assesment of the effectiveness of training relationship enhancement program on increasing intimacy in dual-career couples. Scientific Journal of Kurdistan University of Medical Sciences, 16(4), 37-43.

Etemadi, O., NavvabiNezhad, S., Ahmadi, S., \& Farzad, V. (2006). A study on the effectiveness of cognitive-behaviour couple therapy on couple's intimacy that refers to counseling centers in Esfahan. Psychological Studies, 2(1), 69-87.

Etimadi, O., Amin Jafari, B., \& Shah Seyah, M. (2014). The Impact of Collective Instruction of Couples Based on Relationship Therapy Approach on Increasing Marital Intimacy of the Couples of Shahid Families of the 
City of Isfahan. ISWF. mags, 2(2), 13-38.

Farbod, E., Ghamari, M., \& Majd, M. A. (2014). Investigating the effect of communication skills training for married women on couples' intimacy and quality of life. SAGE Open, 4(2), 1-4. http://dx.doi.org/10.1177/ 2158244014537085

Garmkhani, M. B., Madani, Y., \& Lavasani, M. Q. A. (2014). The effectiveness of cognitive behavioral group training on unrealistic relationship standards and marital intimacy of women. Quarterly Journal of Woman and Society, 5(2), 127-144.

Greeff, P., Hildegarde, L., \& Malherbe, A. (2001). Intimacy and marital satisfaction in spouses. Journal of Sex and Marital therapy, 27(3), 247-257. http://dx.doi.org/10.1080/009262301750257100

Hajian, A., \& Mohammadi, S. (2013). The effect of training solution-focused couples therapy on dimensions of marital intimacy. PakJ Med Sci, 29(1), 321-324. http://dx.doi.org/10.12669/pjms.291(suppl).3525

Hamedi, B., SHafi Abadi, A., Navabinejad, S., \& Delavar, A. (2013). The effectiveness of emotionally focused couple therapy on marital intimacy and self efficacy in students. Educational Administration Research Quarterly, 4(3), 43-56.

Harper, J. M., Schaalje, B. G., \& Sandberg, J. G. (2000). Daily hassles, intimacy, and marital quality in later life marriages. American Journal of Family Therapy, 28(1), 1-18. http://dx.doi.org/10.1080/019261800261770

Hickmon, Jr. W. A., Protinsky, H. O., \& Singh, K. (1997). Increasing marital intimacy: Lessons from marital enrichment. Contemporary family therapy, 19(4), 581-589. http://dx.doi.org/10.1023/A:1026191223476

Hosseini, T., Majd, M. A., \& GHamari, M. (2013). The Effectiveness of Solution-Focused Group Counseling in Enhancing Marital Intimacy of Married Women. Family Health, 1(4), 53-63.

Hosseinian, S., Yazdi, S. M., \& Tabatabaei, M. (2012). The Impact of teaching communication skills on the marital Intimacy of married women. Journal of Cultural Education for Women and Families, 6(18), 46-76.

Isfahani, N. N. (2010). The effect of choice theory training through group learning method on increasing marital intimacy of women. Woman and study of family, 2(8), 105-114.

Isfahani, N. N., Etemadi, A., \& Abadi, A. S. (2013). Effect of meaning-centered training on marital intimacy of women. The Journal of Qazvin University of Medical Sciences, 17(1), 42-47.

Javid, M. M., Soveyzi, R., \& Mousavi, M. R. (2014). The Effectiveness of Skills Training of Improving Marital Relationships on Increasing Intimacy and Personal Well-Being of Women. Journal of Applied Environmental and Biological Sciences, 4(8), 208-216.

Karimi, S., Hasani, M., Soltani, Z., Dalvand, M., \& Zohdi, M. (2012). Effects of Communication Skills Training on Increasing Intimacy between Male Teachers and their Spouses: A Case Study in Baane City. Journal of Basic and Applied Scientific Research, 2(4), 3653-3659.

Kheir, M. G., Givi, H. G., Niloofar, M., \& Shamlo, Z. S. (2013). The effectiveness of intervention based on rational-emotional behavioral therapy in raising intimacy and women's marital satisfaction. Thought and Behavior in Clinical Psychology, 8(29), 27-36.

Kim, M. (2013). The effect of marital intimacy, sex communication, and sexual satisfaction on the marital satisfaction for Korean middle-aged women. Journal of Next Generation Information Technology, 4(8), 441-448.

Lopez, L. M., Hiller, J. E., Grimes, D. A., \& Chen, M. (2012). Education for contraceptive use by women after childbirth. The Cochrane Library, 20(1). http://dx.doi.org/10.1002/14651858.cd001863.pub3

Lopez, L. M., Steiner, M., Grimes, D. A., \& Schulz, K. F. (2013). Strategies for communicating contraceptive effectiveness. The Cochrane Library, 30(4). http://dx.doi.org/10.1002/14651858.cd006964.pub3

Mami, S., Roohandeh, M., \& Kahareh, A. N. (2015). Effectiveness of couple therapy on marital intimacy and life satisfaction in couples's ilam. Indian Journal of Fundamental and Applied Life Sciences, 5(1), 1449-1452.

Martin, G. M., \& Tardif, M. (2014). What we do and don't know about sex offenders' intimacy dispositions. Aggression and Violent Behavior, 19(4), 372-382. http://dx.doi.org/10.1016/j.avb.2014.06.002

Mazlomi, M., Dolatshahi, B., \& Nazari, A. (2012). The effect of enrichment program instruction on enhancement of intimacy among couples with no major conflict. Dena, 5(3/4), 51-62.

McLeod, S. (2008). Cognitive Behavioral Therapy. Simply Psychology. 
Mitchell, A. E. (2007). The effect of self-disclosure and empathic responding on intimacy: Testing an interpersonal process model of intimacy using an observational coding system. (Master's thesis, Texas A\&M University, Texas, United States). Retrieved from http://hdl.handle.net/1969.1/5805

Mohammadi, A., Sohrabi, R., \& Aghdam, G. A. (2013). Effect of narrative therapy on enhancing of couples intimacy. Procedia-Social and Behavioral Sciences, 84(0), 1770-1772. http://dx.doi.org/10.1016/j.sbspro. 2013.07.029

Moreira, H., Crespo, C., Pereira, M., \& Canavarro, M. C. (2010). Marital quality among women with breast cancer: The role of marital intimacy and quality of life. Psicooncologia, 7(7), 61-80.

Motavali, R., Ozgoli, G., Bakhtiari, M., \& Alavimajd, H. (2010). Marital satisfaction and marital Intimacy in employed and unemployed pregnant women of Ardebil City. Journal of Ardabil University of Medical Sciences, 9(4), 315-324.

Nainian, M., \& Nik-Azin, A. (2013). The relationship between intimacy and sexual satisfaction with general health and personal well-being: Investigation of sex and age differences. J Res Behav Sci, 10(7), 735-745.

Nayeri, A., Lotfi, M., \& Noorani, M. (2014). The effectiveness of group training of transactional analysis on intimacy in couples. Procedia-Social and Behavioral Sciences, 152(2014), 1167-1170. http://dx.doi.org/10. 1016/j.sbspro.2014.09.294

Nejad, F. N., Nazari, A. M., \& Bahrainian, A. (2014). Investigating the effect of Training Positive Thinking on Intimacy, Satisfaction and Sexual Performance in Housewives of 5th region in Tehran. Bull. Env. Pharmacol. Life Sci, 3(4), 26-32.

Nejad, F. N., Tork, M., Rad, Z. Z., Nazari, A. M., \& Korivand, B. (2014). The efficacy of positive thinking training on intimacy of housewives in Karaj. Reef Resources Assessment and anagement Technical Paper, 40(1), 420-424.

Oulia, N., Fatehizadeh, M., \& Bahrami, F. (2006). The study of effectiveness of instruction marital enrichment on increasing of marital intimacy. Journal of Family Research, 2(6), 119-135.

Rezaei, J., Ahmadi, S., Etemadi, O., Rezaei, M., Abadi, H., \& Shahdoust, M. (2013). The Effect of Training of Islamic Lifestyle with Emphasis on Family system on Marital Intimacy in Arak. Quarterly Journal of Psychological Studies, 9(3), 79-106.

Salimi, M., \& Fatehizadeh, M. (2012). Investigation of effectiveness of sextual education based on behavioral-cognitive method on sextual intimacy, knowledge and self-expression of married woman in Mobarakeh. Journal of Psychology, 7(28), 111-128.

Shahrestany, M., Doustkam, M., Rahbarda, H., \& Mashhadi, A. (2013). Predicting marital satisfaction over the relational belief and marital intimacy variables in normal and divorced women in Mashhad. Woman and Culture, 4(14), 19-41.

Shakarami, M., Davarniya, R., Zahrakar, K., \& Gohari, S. (2014). The effect of sex education on sexual intimacy of married women. Journal of Nursing Education, 2(1), 34-42.

Shariatzadeh, M., Tabrizi, M., \& Ahghar, G. (2014). The effectiveness of choice theory by grouping method on increasing self-differentiation and intimacy of married students. Knowledge and Health, 8(4), 89-93.

Sharifian, M., Najafi, S., \& Shaghaghi, F. (2011). An Investigation of Couple Communication Program (CCP) on The Life's Quality and Intimacy of Unsatisfied Women. Procedia-Social and Behavioral Sciences, 30, 1991-1994. http://dx.doi.org/10.1016/j.sbspro.2011.10.387

Soltani, A., Molazadeh, J., Mahmoodi, M., \& Hosseini, S. (2013). A study on the effectiveness of emotional focused couple therapy on intimacy of couples. Procedia-Social and Behavioral Sciences, 82(2013), 461-465. http://dx.doi.org/10.1016/j.sbspro.2013.06.293

Taghiyar, Z., Mohammadi, K., \& Zarie, E. (2015). The survey of relationship between sexual satisfaction and marital commitment with marital intimacy in Esfahan couples. South Journal of Educational Psychology and Counseling, 2(1), 44-49.

Tavakol, M., Zarei, E., \& Pour, H. Z. (2014). A comparison between controlling behaviors, intimacy and marital adjustment of Iranian couples resident. Academic Journal of Psychological Studies, 3(7), 415-422.

Uhm, D. C. (2010). Effects on couples' communication, intimacy, conflict and quality of life by foot massage between immigrants. Journal of Korean Academy of Nursing, 40(4), 493-502. http://dx.doi.org/10.4040/ 
jkan.2010.40.4.493

Veshki, S. K., Botlani, S., Shahsiah, M., \& Sharifi, E. (2012). The effect of sex education on marital quality improvment in couples of Qom. Interdisciplinary Journal of Contemporary Research in Business, 4(7), 134-147.

Weinberger, M. I., Hofstein, Y., \& Whitbourne, S. K. (2008). Intimacy in young adulthood as a predictor of divorce in midlife. Personal Relationships, 15(4), 551-557. http://dx.doi.org/10.1111/j.1475-6811.2008. 00215. $\mathrm{x}$

Whisman, M. A., Dixon, A. E., \& Johnson, B. (1997). Therapists' perspectives of couple problems and treatment issues in couple therapy. Journal of Family Psychology, 11(3), 361-366. http://dx.doi.org/10.1037/0893 $-3200.11 .3 .361$

Yoo, H., Bartle-Haring, S., Day, R. D., \& Gangamma, R. (2014). Couple communication, emotional and sexual intimacy, and relationship satisfaction. Journal of Sex and Marital Therapy, 40(4), 275-293. http://dx.doi.org/10.1080/0092623X.2012.751072

Yousefi, N., \& Kiani, M. A. (2014). The Study of Two Psychotherapy Approaches (Rogers Self Theory and Ellis Rational Theory) in Improvement of Bowen Self-differentiation and Intimacy. Iranian Journal of Psychiatry and Behavioral Sciences, 8(1), 32-41.

Zand, M. H., Abadi, A. S., \& Soudani, M. (2013). Effectiveness of Islamic couple therapy on sexuall intimacy among couples attending in Nikan clinic Tehran. Journal of Social Psychology, 7(25), 27-39.

Zarepour, M. (2010). The Effectiveness of Problem Solving Training on Marital Intimacy. (Master student in Family Counseling), University of Tehran, Faculty of Psychology and Education Science, Tehran, Iran.

\section{Copyrights}

Copyright for this article is retained by the author(s), with first publication rights granted to the journal.

This is an open-access article distributed under the terms and conditions of the Creative Commons Attribution license (http://creativecommons.org/licenses/by/3.0/). 